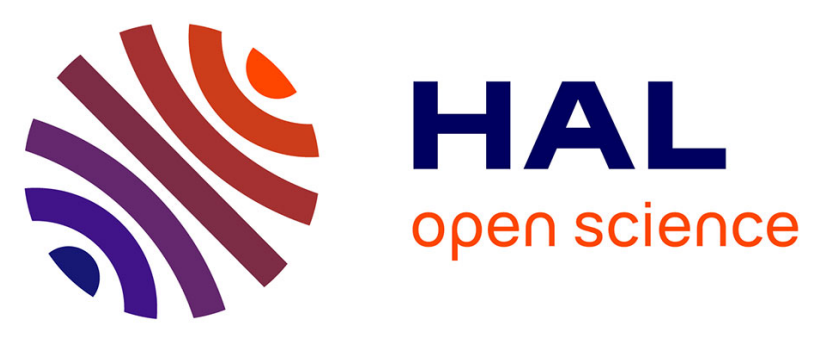

\title{
Les ouvrages de biorétention: synthèse des guides internationaux de conception et de maintenance des filtres plantés pour traitement à la source des eaux de ruissellement urbaines
}

Kelsey Flanagan, Philippe Branchu, Marie-Christine Gromaire

\section{To cite this version:}

Kelsey Flanagan, Philippe Branchu, Marie-Christine Gromaire. Les ouvrages de biorétention: synthèse des guides internationaux de conception et de maintenance des filtres plantés pour traitement à la source des eaux de ruissellement urbaines. Techniques Sciences Méthodes , 2017, 12, pp.89-126. $10.1051 / \mathrm{tsm} / 201712089$. hal-02065428

\section{HAL Id: hal-02065428 \\ https://hal-enpc.archives-ouvertes.fr/hal-02065428}

Submitted on 12 Mar 2019

HAL is a multi-disciplinary open access archive for the deposit and dissemination of scientific research documents, whether they are published or not. The documents may come from teaching and research institutions in France or abroad, or from public or private research centers.
L'archive ouverte pluridisciplinaire HAL, est destinée au dépôt et à la diffusion de documents scientifiques de niveau recherche, publiés ou non, émanant des établissements d'enseignement et de recherche français ou étrangers, des laboratoires publics ou privés. 


\section{Kelsey Flanagan1, Philippe Branchu², Marie-Christine Gromaire1}

${ }^{1}$ LEESU, UMR MA 102, École des Ponts, AgroParisTech, UPEC, UPE, Champs-sur-Marne, 6-8 avenue Blaise Pascal, Cité Descartes, 77455 Marne-la-Vallée Cedex 2, France. kelsey.flanagan@enpc.fr, gromaire@enpc.fr

${ }^{2}$ CEREMA Direction territoriale Ile-de-France, 12 Rue Léon Teisserenc de Bort, 78190 Trappes, France. philippe.branchu@cerema.fr

\section{Mots clés : Biorétention, filtre planté, techniques alternatives, eaux de ruissellements urbaines}

\section{Keywords : Bioretention, Biofiltration, SUDS, urban runoff water}

\section{Résumé :}

Depuis quelques décennies, on observe le développement de systèmes végétalisés conçus pour stocker, filtrer et parfois infiltrer à la source les eaux de ruissellement urbaines. Dans le monde anglophone de nombreux guides ont été publiés afin d'encadrer la conception et la maintenance de ce type d'ouvrage, qualifiés de bioretention. Ces guides, issus de retour d'expérience dans ces pays, proposent des préconisations relatives à l'emplacement des ouvrages, leurs modes de vidange, les caractéristiques des couches du filtre, les critères et méthodes de dimensionnement, le choix des végétaux et l'entretien des ouvrages. Comme ces techniques sont encore relativement peu diffusées en France et ne font pas encore l'objet de tels guides, cet article a pour vocation de dresser une synthèse d'une sélection de ces guides.

\footnotetext{
Abstract:

Over the past few decades, vegetated systems designed for the on-site storage, filtration and sometimes infiltration of urban runoff have been developed and become increasingly popular. Across the English-speaking world, one can find a great many guides providing detailed information as to the design and maintenance of this type of system, referred to as bioretention. These guides, which present advice as to where to locate these systems, how to drain them, the characteristics of the filter media, sizing methods, plant choice and system maintenance, contain a wealth of valuable information and feedback from the experience in these countries. As this technique is relatively new in France and no such detailed guidelines yet exist, this article synthesizes the information from a selection of these guides.
} 


\section{Introduction}

Les eaux de ruissellement urbaines sont contaminées par de nombreux polluants tels que les matières en suspension (MES), les éléments traces métalliques et métalloïdes, ou les micropolluants organiques (Davis and Birch, 2010; Eriksson et al., 2007; Gasperi et al., 2014; Zgheib et al., 2012), dont certains font partie des polluants prioritaires définis par la Directive Cadre sur l'Eau Européenne (European Commission, 2000). Ainsi, la protection de la qualité des milieux récepteurs de cette charge polluante est devenue un objectif prioritaire de la gestion des eaux pluviales.

Plus largement, une prise de conscience des problèmes associés aux modifications du cycle d'eau du fait de l'imperméabilisation des sols lors de l'urbanisation mène à un changement de paradigme en hydrologie urbaine. La stratégie de drainage rapide par des réseaux d'assainissement se voit remplacée par des «techniques alternatives » où les eaux sont gérées au plus proche possible de la source (aussi connu sous les termes de gestion à la source et gestion amont) (Fletcher et al., 2014).

Dans ce contexte, les systèmes végétalisés permettant le stockage, la filtration et éventuellement l'infiltration des eaux de ruissellement urbaines connaissent un succès croissant. Ces ouvrages limitent le flux polluant par l'épuration et la rétention de l'eau (Davis, 2007; DiBlasi et al., 2009; Hatt et al., 2009). En outre, ils peuvent limiter les effets hydrologiques de l'urbanisation d'un site en réduisant le débit de pointe, le volume ruisselé en favorisant l'infiltration et l'évapotranspiration (Davis, 2008; Hatt et al., 2009; Heasom et al., 2006), tout en contribuant au paysage et à la biodiversité de l'espace urbain (Kazemi et al., 2011).

Plus spécifiquement, des ouvrages végétalisés pour la gestion en amont des eaux de ruissellement urbaines avec un objectif de filtration apparaissent en France sous des appellations de noue de voirie (Leroy et al., 2016), de noue filtrante (Servier, 2016), de fossé filtrant et de filtre planté (Ramier et al., 2016). Bien qu'il existe des prescriptions générales pour l'infiltration de l'eau en France (Ecopluies, 2009; MGD Infiltration, 2006), ces ouvrages de traitement, conçus de manière empirique, ne bénéficient, à l'heure actuelle, d'aucun guide détaillé de conception.

Ces ouvrages végétalisés de filtration existe depuis quelques décennies à l'international. En effet, depuis la publication du premier guide sur la technique dite de bioretention en 1993 au Maryland aux États-Unis (Roy-Poirier et al., 2010), cette technique s'est propagée à travers le monde anglophone. Elle fait maintenant l'objet d'un grand nombre de guides de conception et de maintenance, le plus fréquemment sous l'appellation de bioretention, mais aussi sous la désignation de biofiltration. Ces guides présentent des recommandations détaillées par rapport à l'emplacement des ouvrages, aux matériaux de construction, aux précautions à prendre afin de minimiser les risques associés aux ouvrages, aux méthodes de dimensionnement et aux pratiques de maintenance à prévoir. Bien que provenant de contextes variés et parfois très différents de la France, ils constituent un retour d'expérience important, soulignant les bonnes pratiques à adopter et les pièges à éviter lors de la mise en place et de l'opération des ouvrages de biorétention. L'objectif de cet article est ainsi de synthétiser et d'analyser le contenu d'une sélection de ces guides, représentant une variété de contextes.

\section{Définition des dispositifs de biorétention}

Un dispositif de biorétention (Figure 1) consiste en une dépression végétalisée remplie d'un substrat filtrant vers laquelle les eaux de ruissellement d'une surface imperméabilisée (ou partiellement) sont dirigées, puis stockées, filtrées et éventuellement infiltrées. Après stockage en surface, l'eau est filtrée à travers le substrat, choisi pour sa perméabilité et sa capacité de dépolluer l'eau. Dans certains cas, le substrat de filtration est recouvert par un paillage. L'eau infiltrée peut être collectée par un drain en fond d'ouvrage et/ou sortir du système par exfiltration (percolation ou absorption de l'eau dans le sol naturel sous-jacent). Dans le cas où un drain est présent, il est généralement installé dans une couche de drainage en gravier, séparée de la couche de substrat filtrant par une couche de transition de granulométrie intermédiaire. Il existe des configurations où le drain est surélevé pour créer un niveau saturé permanent. Dans certains cas, l'ouvrage peut être étanche pour empêcher l'infiltration. (Clar et al., 2004; Roy-Poirier et al., 2010; LeFevre et al., 2014; Liu et al., 2014).

Dans ce type de système, les processus naturels associés à l'écosystème du sol assurent la rétention des eaux de ruissellement ainsi que leur dépollution. Le devenir des polluants sera influencé notamment par les processus de filtration physique des polluants particulaires, sorption des polluants dissous, volatilisation, biodégradation et l'interaction avec la végétation (accumulation par les plantes ou stabilisation dans la zone racinaire) (LeFevre et al., 2014).

Il existe deux concepts voisins de biorétention : les filtres plantés à roseaux ou zones humides artificielles (constructed wetlands) et les jardins de pluie. On peut distinguer les ouvrages de biorétention des filtres plantés à roseaux par les conditions hydrologiques typiques ainsi que la végétation retrouvée. En effet, au contraire d'une zone humide artificielle, un ouvrage de biorétention alimenté par les eaux de ruissellement n'est généralement pas couplé à un bassin de stockage en amont et est conçu pour se vider dans les heures suivant un orage. Son sol est rarement saturé, pouvant même devenir très sec entre les événements pluvieux ; la végétation d'une zone humide n'est donc pas adaptée, il faudrait plutôt la végétation d'une zone d'humidité variable, telle qu'une forêt (Roy-Poirier et al., 2010). En anglais, le terme rain garden, est parfois utilisé comme un synonyme de biorétention (Dietz and Clausen, 2005; Le Coustumer et al., 2012), mais peut se référer également à une variante plus petite et moins technique (CIRIA, 2015; FAWB et al., 2009). En France, une fiche technique 
diffusée par le Certu définit un jardin de pluie comme « une dépression plantée, créée pour récupérer, autant que possible, l'excès de ruissellement d'une maison ou d'un autre bâtiment et de son environnement paysager » (Le Nouveau, 2010), ce qui ressemble effectivement à un ouvrage de biorétention sans la notion d'un substrat filtrant spécifique.

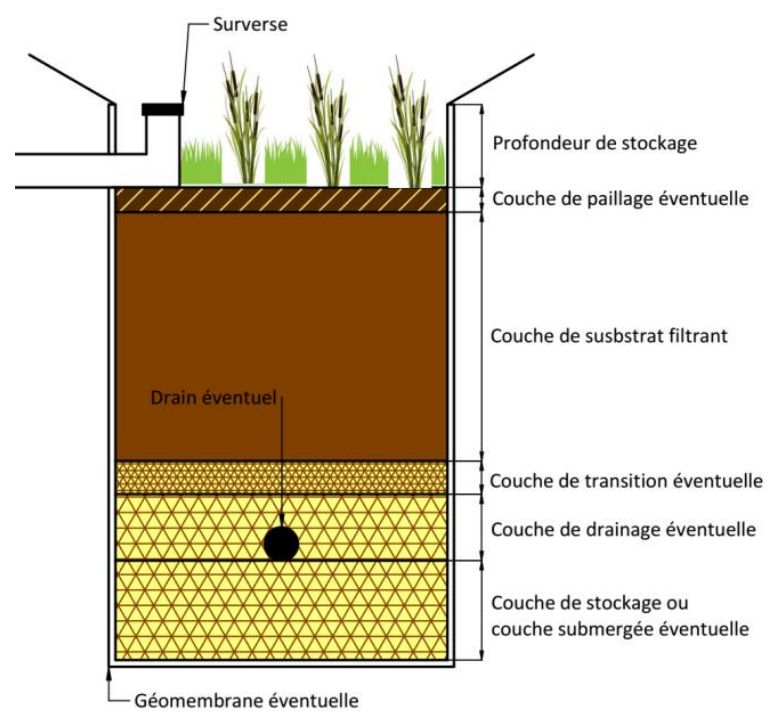

Figure 1 : Un dispositif de biorétention générique

\section{Présentation des guides étudiés}

Dans cet article, nous étudions une sélection de guides de conception et de maintenance des ouvrages de biorétention. Lors de la sélection, nous avons retenu, tout d'abord, les guides généralement considérés comme faisant-référence dans le domaine ou provenant des territoires où l'activité de recherche scientifique est importante sur ces pratiques (FAWB, 2009; NCDWQ, 2017; PGCDER, 2007). D'autres guides ont été choisis afin de souligner des approches de conception originales (UWWRI, 2006) ou des contextes et/ou des pays différents (CIRIA, 2015; CVC, 2010; NSC, 2008; PUB, 2011; UDFCD, 2015a). Ces guides sont applicables à une variété d'échelles, depuis l'échelle nationale (CIRIA, 2015; PUB, 2011) à l'échelle locale (CVC, 2010; NSC, 2008; PGCDER, 2007; UDFCD, 2015a) en passant par l'échelle d'un état aux EtatsUnis (NCDWQ, 2017; UWWRI, 2006). Le guide en provenance de Melbourne est le produit d'un programme de recherche qui n'est pas applicable à une région particulière (FAWB, 2009). Ces guides ne sont généralement pas réglementaires mais sont des outils d'aide aux acteurs de développement urbain pour l'implémentation des stratégies de gestion à la source des eaux pluviales.

Comme les ouvrages de biorétention sont conçus afin de répondre à une problématique hydrologique, elle-même conditionnée par le contexte climatique local, une prise en compte de ce contexte apparaît comme nécessaire pour l'interprétation des guides. Ainsi, on présente dans le Tableau I les caractéristiques climatiques associées aux neuf guides étudiés et, pour comparaison, celles associées aux trois plus grandes villes françaises.

On note que la majorité des guides, et notamment les guides «faisant-référence », proviennent de climats tempérés sans saison sèche (CIRIA, 2015; FAWB, 2009; NCDWQ, 2017; NSC, 2008; PGCDER, 2007), pouvant correspondre aux zones climatiques de Paris et de Lyon. La répartition de la pluie sur toute l'année est plutôt favorable aux systèmes de biorétention, les flux à traiter étant répartis sur une période plus longue, et ce climat limitant les problèmes de stress hydrique des plantes par rapport à un climat méditerranéen. De plus, compte tenu des températures douces, le sol n'est pas susceptible de geler sur de longues périodes en hiver ni d'être soumis à un cumul important de neige comme en climat de montagne - le système fonctionne tout au long de l'année, même si les plantes sont moins développées en hiver. Les autres guides permettent de traiter d'autres conditions climatiques : un climat équatorial avec une pluviométrie très élevée à Singapour, des climats continentaux, plus froids, à Madison (Etats-Unis) et à Toronto (Canada) et un climat de steppe-aride à Denver (Etats-Unis).

En rapport avec ce zonage climatique, les régions étudiées diffèrent de par l'intensité et la fréquence des événements pluvieux. Ces différences sont illustrées en Tableau I par les cumuls de pluie moyens journaliers $\left(\mathrm{H}_{\text {pluie par jour }}\right)$ et par les hauteurs de pluie de périodes de retour de 1 an et de 10 ans pour des durées de $1 \mathrm{~h}$ et $24 \mathrm{~h}$. Tout comme Londres, Paris est caractérisé par des pluies fréquentes d'ampleur et d'intensité faible. A Lyon, la pluie est moins fréquente et d'ampleur et d'intensité plus importante, cette situation est encore plus exacerbée à Marseille. Cependant, les intensités de pluie en France restent généralement plus faibles que celles observées sur la côte est de 1'Amérique du Nord et à Singapour. 


\begin{tabular}{|c|c|c|c|c|c|c|c|c|c|c|}
\hline$\frac{1}{\frac{0}{3}}$ & 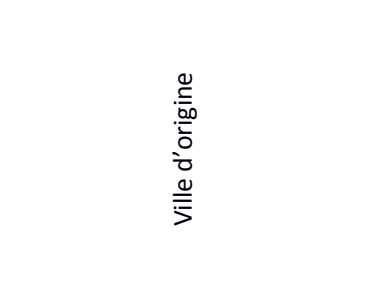 & 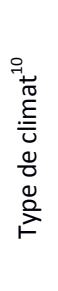 & 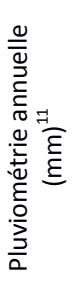 & 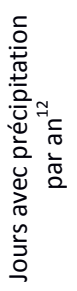 & 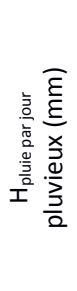 & 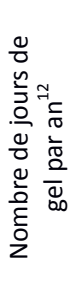 & 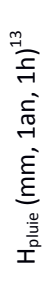 & 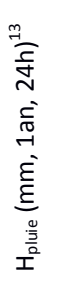 & 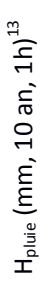 & 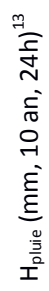 \\
\hline 1 & Melbourne, Australie & $\mathrm{Cfb}$ & 657 & 144 & 4.6 & 2 & 14 & 51 & 26 & 96 \\
\hline \multirow{2}{*}{2} & \multirow{2}{*}{$\begin{array}{l}\text { North Shore City, } \\
\text { Nouvelle-Zélande }\end{array}$} & \multirow{2}{*}{$\mathrm{Cfb}$} & \multirow{2}{*}{1160} & \multirow{2}{*}{172} & \multirow{2}{*}{6.7} & \multirow{2}{*}{0} & \multicolumn{2}{|c|}{$\mathrm{T}=1,58$ ans } & 37 & 126 \\
\hline & & & & & & & 17 & 108 & & \\
\hline 3 & $\begin{array}{c}\text { Upper Marlboro, } \\
\text { Maryland, Etats-Unis }\end{array}$ & Cfa & 1028 & 106 & 9.7 & 105 & 31 & 69 & 55 & 131 \\
\hline 4 & $\begin{array}{c}\text { Raleigh, Caroline du Nord, } \\
\text { Etats-Unis }\end{array}$ & Cfa & 1150 & 101 & 11.4 & 61 & 37 & 76 & 62 & 139 \\
\hline 5 & Madison, Wisconsin, Etats-Unis & $\mathrm{Dfb}$ & 940 & 115 & 8.2 & 143 & 24 & 63 & 50 & 103 \\
\hline 6 & Denver, Colorado, Etats-Unis & BSk & 360 & 87 & 4.1 & 157 & 17 & 39 & 33 & 72 \\
\hline \multirow{2}{*}{7} & \multirow{2}{*}{ Toronto, Canada } & \multirow{2}{*}{$\mathrm{Dfb}$} & \multirow{2}{*}{859} & \multirow{2}{*}{146} & \multirow{2}{*}{5.9} & \multirow{2}{*}{148} & \multicolumn{2}{|c|}{$\mathrm{T}=2$ ans } & 40 & 80 \\
\hline & & & & & & & 23 & 48 & & \\
\hline \multirow{2}{*}{8} & \multirow{2}{*}{ Londres, Angleterre } & \multirow{2}{*}{$\mathrm{Cfb}$} & \multirow{2}{*}{760} & \multirow{2}{*}{236} & \multirow{2}{*}{3.2} & \multirow{2}{*}{64} & \multicolumn{2}{|c|}{$\mathrm{T}=2$ ans } & 26 & 57 \\
\hline & & & & & & & 8 & 29 & & \\
\hline \multirow[t]{4}{*}{9} & Singapore, Singapore & Af & 2279 & 218 & 10.5 & 0 & 50 & 77 & 93 & 144 \\
\hline & Paris, France & $\mathrm{Cfb}$ & 648 & 193 & 3.4 & 50 & 18 & 33 & 32 & 61 \\
\hline & Lyon, France & $\mathrm{Cfb}$ & 735 & 116 & 6.3 & 56 & 19 & 54 & 33 & 85 \\
\hline & Marseille, France & Csa & 576 & 92 & 6.3 & 26 & 26 & 82 & 45 & 110 \\
\hline
\end{tabular}

Tableau I : Liste des climats associés aux guides et aux villes françaises. ${ }^{1}\left(\right.$ FAWB, 2009), ${ }^{2}(\mathrm{NSC}, 2008),{ }^{3}(\mathrm{PGCDER}, 2007),{ }^{4}(\mathrm{NCDWQ}, 2017),{ }^{5}(\mathrm{UWWRI}$, 2006), ${ }^{6}(\mathrm{UDFCD}, 2015 \mathrm{a}),{ }^{7}(\mathrm{CVC}, 2010),{ }^{8}$ (CIRIA, 2015), ${ }^{9}(\mathrm{PUB}, 2011),{ }^{10} \mathrm{Af}=$ climat équitorial, BSk= climat de steppe semi-aride, sec et froid , Cfa $=$ climat tempéré sans saison sèche, été chaud, $\mathrm{Cfb}=$ climat tempéré sans saison sèche, été tempéré, Csa= climat tempéré avec été sec et chaud, Dfb= climat continental sans saison sèche, été tempéré (Kottek et al., 2006), ${ }^{11}$ (Peel et al., 2007), ${ }^{12}$ (Canty et al., 2016), ${ }^{13}$ Données Australie (Australian Government, 2016), New Zealand (NIWA, 2016), Etats-Unis (US Department of Commerce,2016), Canada (Government of Canada, 2015), UK (Centre for Ecology and Hydrology, 2016), Singapore (Qingyu et al., 2012), Paris de Météo France, Lyon (Communauté de Lyon Direction de l'eau, 2012), Marseille (Préfet des Bouches-du-Rhône, 2015) et (Egis Eau, 2011).

\section{Synthèse des guides}

\subsection{Recommandations en terme de type de bassin versant d'apport, d'emplacement de l'ouvrage et de mode d'alimentation}

Les ouvrages de biorétention s'insèrent dans un environnement urbain, où ils reçoivent les eaux de ruissellement d'un bassin versant d'apport qu'ils traitent avant de les évacuer vers le sous-sol ou vers un réseau de drainage. Un premier type de consigne fréquemment relevé dans les guides concerne la relation de l'ouvrage avec son environnement: les caractéristiques acceptables du bassin versant d'apport, l'emplacement des ouvrages, les modes d'alimentation et les besoins en prétraitement.

Les caractéristiques des bassins versants d'apport considérés incluent la surface, la pente et l'occupation du sol. La biorétention étant conçue comme une technique de gestion en amont des eaux de ruissellement, la surface du bassin versant d'apport est généralement limitée à une valeur maximale qui varie entre 0,4 ha (UDFCD, 2015a) et 0,8 ha (CIRIA, 2015; CVC, 2010; PGCDER, 2007). Seul le guide australien élargit le domaine d'application en intégrant également des ouvrages en aval des réseaux d'assainissement, pouvant avoir un bassin versant supérieur à 50 ha (FAWB, 2009).

La pente du bassin versant est également prise en compte, limitée à 20\% (NSC, 2008; PGCDER, 2007), elle se situe idéalement entre 1 et 5\% (CVC, 2010). L'ouvrage doit quant à lui avoir une surface complètement plane afin d'assurer une bonne répartition des flux d'infiltration. Une pente initiale importante peut engendrer des coûts de terrassement importants (CIRIA, 2015; PGCDER, 2007; UDFCD, 2015a). De plus, en zone pentue, l'écoulement rapide du ruissellement peut engendrer une érosion du sol, aussi bien sur le bassin versant d'apport (UDFCD, 2015a) que dans l'ouvrage lui-même (FAWB, 2009). L'effet de la pente est exacerbé dans le cas des ouvrages linéaires, par exemple en bordure de route, pour lesquels on recommande la séparation de l'ouvrage en biefs et une pente maximale de 5\% (FAWB, 2009).

On constate également des limitations en termes d'occupation du sol du bassin versant afin de limiter l'apport de polluants ou de particules. En région aride, il est conseillé d'éviter d'implanter ces ouvrages sur des bassins versants peu végétalisés, susceptibles de générer des quantités importantes de sol érodé (UDFCD, 2015a). Dans les climats froids, des restrictions d'usage de la biorétention pour des voiries connaissant des épandages fréquents de sel (CVC, 2010) ou de sable (UDFCD, 2015a) en période de viabilité hivernale sont notées. Plusieurs guides soulignent que l'existence de travaux sur le bassin versant peut générer des problèmes de colmatage dû à l'apport de particules ou au compactage du substrat en cas de passage 
des engins de construction. Ainsi, il est conseillé d'attendre la fin des travaux pour sa mise en service (CIRIA, 2015; CVC, 2010; PUB, 2011; UDFCD, 2015a).

L'emplacement du système de biorétention au sein du bassin versant est également un élément important de sa conception. Afin de minimiser les travaux de terrassement, il est recommandé que la topographie naturelle du site soit préservée si possible et que l'ouvrage soit placé au point bas du bassin versant (PGCDER, 2007). Pour des ouvrages non-étanches, l'infiltration d'eau peut poser des risques vis-à-vis de l'environnement construit. Deux approches sont proposées pour gérer ce risque: obligation d'obtention de l'avis d'un ingénieur géotechnique (UDFCD, 2015a) ou respect d'une distance minimale entre l'ouvrage et tout bâtiment, allant de 1,5 m (PGCDER, 2007) à $4 \mathrm{~m}$ (NSC, 2008). Une ultime contrainte souvent citée est le respect d'une distance minimale entre le fond de l'ouvrage et le niveau maximal de la nappe phréatique ; cette distance varie de 0,6 m (NCDWQ, 2017; PGCDER, 2007) à 1 m (CIRIA, 2015; CVC, 2010) ; pour comparaison, un guide français le limite également à $1 \mathrm{~m}$ (Ecopluies, 2009).

Comme tout ouvrage de gestion des eaux pluviales, la capacité hydraulique d'un système de biorétention peut être dépassée en cas d'événements pluvieux plus importants que celui associé au dimensionnement. Deux approches sont proposées, la première, considérée préférable par deux guides, consiste à dériver l'eau avant son arrivée dans l'ouvrage (by-pass) lorsque ce dernier est plein (PGCDER, 2007), la deuxième fait intervenir un dispositif de surverse (CIRIA, 2015; CVC, 2010; FAWB, 2009; NCDWQ, 2017; NSC, 2008; PGCDER, 2007; UDFCD, 2015a; UWWRI, 2006). La surverse doit alors être la plus proche possible de l'entrée pour minimiser les phénomènes d'érosion du substrat (PUB, 2011).

Les ouvrages de biorétention peuvent être alimentés par ruissellement superficiel ou par un écoulement canalisé. En cas d'écoulement canalisé, il est souvent considéré nécessaire de prévoir un dispositif de dissipation d'énergie tel que un forebay ou bief amont (CIRIA, 2015), des enrochements ou de la végétation dense (CVC, 2010; NSC, 2008; PUB, 2011) pour éviter l'érosion du sol de l'ouvrage. Pour éviter ce problème, l'alimentation par ruissellement superficiel est souvent favorisée (FAWB, 2009; NCDWQ, 2017; NSC, 2008).

Il est souvent considéré comme nécessaire de prétraiter le ruissellement arrivant afin de retenir une partie des particules et d'éviter le colmatage de l'ouvrage. Certains guides recommandent un prétraitement limité aux bassins versants les plus pollués (NSC, 2008), susceptibles d'apporter beaucoup de particules ou de déchets (CIRIA, 2015; CVC, 2010; FAWB, 2009; UDFCD, 2015a) ou de taille importante (FAWB, 2009; NSC, 2008; PUB, 2011; UDFCD, 2015a), alors que d'autres prévoient systématiquement un prétraitement (NCDWQ, 2017; PGCDER, 2007).

\subsection{Modes de vidange de l'ouvrage}

Il existe différents modes de vidange des ouvrages de biorétention, qui se déclinent en fonction des objectifs assignés à l'ouvrage et des contraintes liées à son implantation. La Figure 2 montre une typologie des ouvrages de biorétention en fonction des modes d'évacuation possibles.

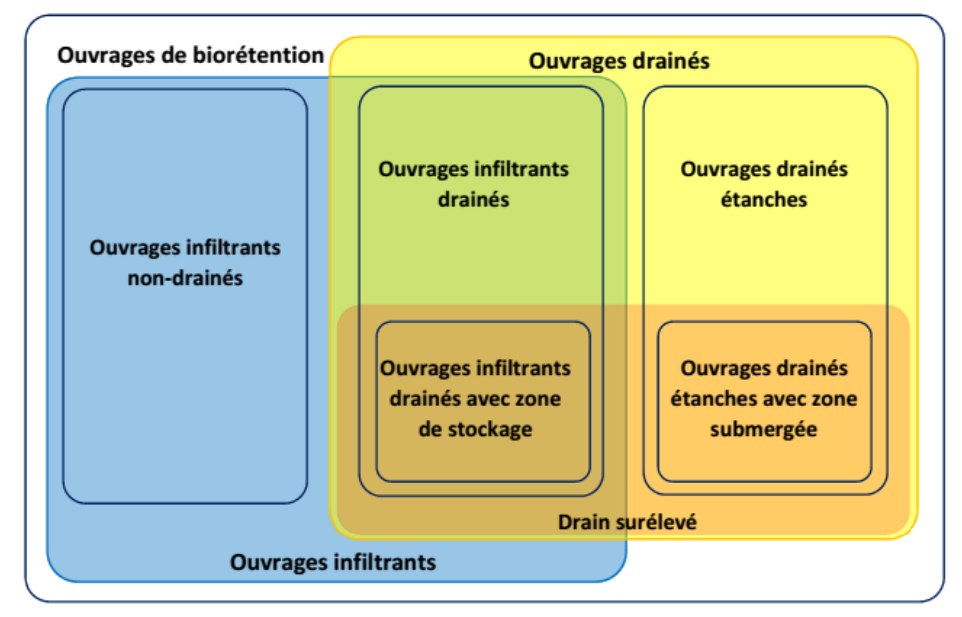

Figure 2 : Configuration possibles de drainage des ouvrages de biorétention

Il est tout d'abord possible de distinguer les ouvrages infiltrants, où au moins une partie de l'eau s'infiltre dans le sol sousjacent, et les ouvrages étanches, non-infiltrants. Cette distinction est présente dans tous les guides, mais différents raisonnements la motivent. En Australie et à Singapour, les ouvrages de biorétention sont recommandés pour la récupération et le prétraitement des eaux pluviales avant une réutilisation (FAWB, 2009; PUB, 2011). Dans ce cas, l'ouvrage est étanchéifié afin de maximiser la proportion d'eau récupérée. L'étanchéification est également recommandée lorsqu'il existe un enjeu de protection de la nappe phréatique ou des infrastructures (CVC, 2010; FAWB, 2009; NSC, 2008; PGCDER, 2007; UDFCD, 2015a). Deux guides affirment que l'étanchéification s'avère nécessaire uniquement si les enjeux ci-dessus sont présents et si la conductivité hydraulique du sol sous-jacent est supérieure à $10 \%$ de la conductivité hydraulique du substrat de l'ouvrage. Dans le cas d'une conductivité plus faible, il est supposé que le fonctionnement du 
drain rend l'étanchéification non nécessaire (CIRIA, 2015; PUB, 2011). Deux guides ne mentionnent pas la possibilité d'étanchéifier le système et le conçoivent uniquement comme un système infiltrant (NCDWQ, 2017; UWWRI, 2006).

Une autre distinction au sein des ouvrages de biorétention s'appuie sur la présence ou non de drains. Si tous les systèmes étanches sont drainés, certains ouvrages non-étanches le sont également. Un drain est généralement recommandé dans un ouvrage non-étanche lorsque le sol sous-jacent n'est pas suffisamment perméable pour assurer le temps de vidange envisagé (CIRIA, 2015; CVC, 2010; FAWB, 2009; NCDWQ, 2017; NSC, 2008; PGCDER, 2007; PUB, 2011; UDFCD, 2015a; UWWRI, 2006).

L'emplacement du drain au sein de l'ouvrage peut également être un élément de discrimination. En effet, le drain (ou la sortie de drain) peut être situé au fond de l'ouvrage ou surélevé, ce qui créé une zone non-drainée. Cette zone répond à des objectifs différents selon que l'ouvrage est ou non étanché. Pour les ouvrages étanches, la zone de stockage restera remplie entre les événements pluvieux. Pendant cette période, la zone peut devenir anoxique, ce qui permet les processus anaérobie de dénitrification (FAWB, 2009; PGCDER, 2007; PUB, 2011). Elle sert de plus de réserve hydrique pour la végétation en périodes sèches (FAWB, 2009; PUB, 2011). Dans le cas des ouvrages non-étanches, la zone de stockage créée par un drain surélevé permet d'augmenter la proportion d'eau infiltrée par rapport à un système drainé classique (CIRIA, 2015; NSC, 2008; PGCDER, 2007; UWWRI, 2006). On retrouve là aussi l'idée qu'un drain surélevé peut encourager la dénitrification en créant une zone anaérobie (NCDWQ, 2017; PGCDER, 2007). Un système intermédiaire est également proposé : nonétanche au niveau de la couche de filtration, avec le drain surélevé et étanche au niveau de la zone de stockage afin de garder une réserve d'eau pour la végétation dans les climats secs (FAWB, 2009; PUB, 2011)

Les guides s'intéressent également aux matériaux de drainage. Le drain est généralement installé dans une couche de drainage en gravier. Selon le CIRIA (2015), il doit posséder une conductivité hydraulique supérieure à celle du sol et doit être surdimensionné pour les écoulements attendus afin qu'il ne limite pas l'écoulement dans le système. Un guide suggère l'installation d'un drain avec un orifice ajustable et accessible pour contrôler le débit à la sortie, afin de pouvoir optimiser le fonctionnement hydrologique du système (UWWRI, 2006). Il peut s'avérer utile de prévoir un regard sur le drain, ce qui permet son inspection et son nettoyage (CVC, 2010; NCDWQ, 2017). Certains guides recommandent l'utilisation d'un tuyau perforé en polychlorure de vinyle (PVC) (FAWB, 2009) ou en polyéthylène haute densité (PEHD) (CVC, 2010), alors que d'autres affirment qu'un drain agricole serait également acceptable (NSC, 2008; PGCDER, 2007).

\subsection{Couches du filtre}

Les ouvrages de biorétention les plus simples sont constituées d'une unique couche de substrat filtrant, mais certains ouvrages comportent d'autres couches: une couche de paillage, une couche de transition, une couche de drainage et une couche de stockage ou submergée (Figure 1). Toutes ces couches font l'objet de recommandations dans les guides (Tableau II).

\begin{tabular}{|c|c|c|c|c|c|c|}
\hline \multicolumn{2}{|c|}{ Couche } & Paillage & $\begin{array}{c}\text { Couche de substrat } \\
\text { filtrant }\end{array}$ & $\begin{array}{l}\text { Couche de } \\
\text { transition }\end{array}$ & $\begin{array}{c}\text { Couche de } \\
\text { drainage }\end{array}$ & Couche de stockage \\
\hline \multirow{2}{*}{\multicolumn{2}{|c|}{ Matériaux }} & copeaux de bois & cf. Figure 3 & $\begin{array}{l}\text { sable ou } \\
\text { aravier fin }\end{array}$ & gravier & $\begin{array}{c}\text { gravier ou sable, } \\
\text { possiblement avec } \\
\text { une source de }\end{array}$ \\
\hline & & $x$ & $30-50^{a}, 50-70^{b}$ & 5 & 15 & $30 *$ \\
\hline \multirow{8}{*}{ 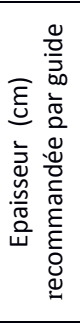 } & 2 & $5-7.5$ & $60-100$ & 10 & $20-30$ & $30 *$ \\
\hline & 3 & $7.6-10.2$ & $76-102$ & $8-20$ & $\circ$ & $<30^{*}$ \\
\hline & 4 & $7.6-10.2$ & $>46^{a, c},>60^{b, c},>90^{d}$ & 10 & 18 & 30 \\
\hline & 5 & - & - & - & - & $<122^{*}$ \\
\hline & 6 & $\mathrm{x}$ & $>46$ & - & 15 & - \\
\hline & 7 & 7.5 & $100-125^{\mathrm{e}},>100^{\mathrm{d}},>50^{f}$ & 10 & 20 & $>10^{*}$ \\
\hline & 8 & $<7.5^{*}$, parfois $\mathrm{x}$ & $75-100^{\mathrm{e}},>40^{\mathrm{f}}$ & $>10$ & $>10$ & ${ }^{\circ *}$ \\
\hline & 9 & - & $>60^{e},>100^{d},>40^{f}$ & $>10$ & $>20$ & $>30 \mathrm{~cm}^{*}$ \\
\hline
\end{tabular}

Tableau II : Matériaux et épaisseurs des couches du filtre. ${ }^{1}\left(\right.$ FAWB, 2009), ${ }^{2}(\mathrm{NSC}, 2008),{ }^{3}\left(\right.$ PGCDER, 2007), ${ }^{4}(\mathrm{NCDWQ}, 2017),{ }^{5}($ UWWRI, 2006),

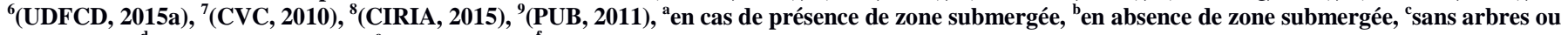
arbustes, ${ }^{\mathrm{d}}$ si arbres ou arbustes, ${ }^{\mathrm{e}}$ gamme idéale, ${ }^{\mathrm{f}}$ gamme acceptable mais non-idéale, * : la couche est optionnelle, - :absence de recommandation, ${ }^{\circ}$ :absence de précision de profondeur, $x$ : couche est contre-indiquée

L'utilisation du paillage ne fait pas consensus. Si certains guides présentent le paillage, généralement une couche d'environ $7,5 \mathrm{~cm}$ de bois râpé ou de copeaux de bois, comme une partie essentielle d'un système de biorétention (CVC, 2010; NCDWQ, 2017; NSC, 2008; PGCDER, 2007), d'autres affirment qu'il doit être évité (CIRIA, 2015; FAWB, 2009; UDFCD, 2015a). D'un côté, il contribue à la filtration et sorption des polluants et joue un rôle de prétraitement, protégeant le substrat du colmatage. Son remplacement, à effectuer régulièrement, permet d'enlever une grande partie du sédiment cumulé dans l'ouvrage (NCDWQ, 2017; PGCDER, 2007). De l'autre, il peut flotter, bloquant le dispositif de surverse (CIRIA, 2015; FAWB, 2009; UDFCD, 2015a). Un des partisans du paillage recommandent de concevoir les systèmes de biorétention en dérivation, de telle sorte qu'un dispositif de surverse n'est pas nécessaire, minimisant ainsi le risque, et l'un recommande la mise en place d'une couverture anti-érosion pour éviter la flottation du paillage (PGCDER, 2007). 
Sous la couche de paillage éventuelle se trouve la couche du substrat filtrant, dont l'épaisseur recommandée est précisée par chaque guide (cf. Table 2). On retrouve une valeur minimale recommandée de $30 \mathrm{~cm}$ applicable uniquement au cas d'un système avec une zone de stockage au fond (FAWB, 2009). La plupart des valeurs se situent dans la gamme de $50 \mathrm{~cm}$ à 100 $\mathrm{cm}$. Certains guides autorisent une épaisseur plus faible en cas de contraintes de profondeur (CVC, 2010; PUB, 2011). Le guide du Royaume Uni autorise une épaisseur plus faible pour de petits ouvrages (CIRIA, 2015). Certains guides proposent d'adapter l'épaisseur du substrat au type de végétation (CVC, 2010; NCDWQ, 2017; PUB, 2011). La nature de ce substrat fait l'objet d'un grand nombre de recommandations, détaillées dans la section suivant.

Les autres couches pouvant être recommandées dans un ouvrage de biorétention sont celles associées à son drainage et le stockage éventuel sous le drain. Le drain est généralement installé au sein d'une couche de gravier d'une épaisseur de 10-30 cm (CVC, 2010; FAWB, 2009; NCDWQ, 2017; NSC, 2008; PGCDER, 2007; PUB, 2011; UDFCD, 2015a), la couche de drainage.

Afin d'éviter le lessivage du substrat filtrant dans la couche de drainage, ces deux couches sont généralement séparées par une couche de transition d'un matériau de granulométrie intermédiaire et d'une épaisseur de 5-20 cm (CVC, 2010; FAWB, 2009; NCDWQ, 2017; NSC, 2008; PGCDER, 2007; PUB, 2011). Le guide australien suggère un critère de chevauchement pour choisir la granulométrie de la couche de transition par rapport aux couches de substrat filtrant et de drainage :

$D_{15}$, couche de transition $\leq 5 \cdot D_{85}$, couche de substrat filtrant et $D_{15, \text { couche de drainage }} \leq 5 \cdot D_{85}$, couche de transition

où $D_{15}$ est le $15^{\text {eme }}$ percentile des diamètres des particules et $D 85$ est le $85^{\text {eme }}$ percentile.

Ce critère assure que les plus petites particules de la couche plus grossière et les plus grandes particules de la couche plus fine aient une taille du même ordre de grandeur. Ce guide déconseille l'utilisation d'un géotextile pour séparer les couches de substrat filtrant et de drainage du fait d'un risque de colmatage (FAWB, 2009). Dans le guide du CIRIA (2015), il est affirmé que malgré les observations de colmatage en Australie, un géotextile en adéquation avec le sol ne devrait pas poser de problème de colmatage et le guide de NCDEQ (2017) permet un géotextile quand le bassin versant génère peu de particules.

La dernière couche pouvant être présente dans un ouvrage de biorétention est la couche de stockage. En général, cette couche est constituée du même matériel que la couche de drainage, du gravier. Les épaisseurs recommandées varient de 10 cm (CVC, 2010) à $122 \mathrm{~cm}$ (UWWRI, 2006), la valeur la plus fréquemment citée est de $30 \mathrm{~cm}$ (FAWB, 2009; NSC, 2008; PGCDER, 2007; PUB, 2011). Deux guides recommandent l'ajout de matière organique à cette couche afin de favoriser les processus de dénitrification (FAWB, 2009; PUB, 2011).

\subsection{Propriétés du substrat filtrant}

Le substrat de filtration, et ses propriétés, constituent un élément clef de l'ouvrage, tant sur le plan hydraulique qu'épuratoire. La prise en compte du substrat comme un objet de conception implique l'excavation et le remplacement du sol en place. Elle permet alors de distinguer les systèmes de biorétention des techniques alternatives conçues uniquement pour le contrôle quantitatif de l'eau tels que les bassins d'infiltration ou les noues enherbées traditionnelles. Chaque guide présente ainsi des recommandations détaillées pour les caractéristiques du substrat. Même si les guides ne citent pas toujours les mêmes paramètres, on trouve fréquemment des recommandations concernant la conductivité hydraulique, la granulométrie, la proportion de matière organique, le $\mathrm{pH}$ et la teneur en nutriments du substrat de filtration. Le seul guide ne comportant pas de recommandations de ce type est celui du Wisconsin qui préconise que le sol en place soit caractérisé et remplacé uniquement si sa conductivité hydraulique est très faible, ce qui impliquerait un ouvrage très grand. Dans ce cas, son remplacement peut être envisagé si le coût des travaux ne surpasse pas le coût d'un ouvrage plus grand (UWWRI, 2006).

La conductivité hydraulique ( $K_{s}$, Figure 3 ) détermine la vitesse de percolation de l'eau dans l'ouvrage. Ainsi, un $K_{s}$ élevé permet de minimiser la surface d'ouvrage nécessaire pour évacuer un volume d'eau donné. Cependant, un $K_{s}$ élevé conduit également à réduire le temps de séjour de l'eau dans le substrat, ce qui est limitant vis à vis de la rétention de polluants dans l'ouvrage. Il est aussi souhaitable, pour la survie des plantes en période sèche, que le substrat retienne de l'eau, ce qui est favorisé par un $K_{s}$ plus faible. Il est donc nécessaire de choisir une conductivité hydraulique permettant un équilibre entre ces différents facteurs. La plupart des guides précisent des gammes acceptables de $K_{s}$ (CIRIA, 2015; FAWB, 2009; NCDWQ, 2017; NSC, 2008; PUB, 2011). Deux guides proposent uniquement une valeur minimale de $K_{s}$ (CVC, 2010; PGCDER, 2007). Les $K_{s}$ recommandés par les guides se trouvent dans la gamme de $3,5 \cdot 10^{-6} \mathrm{~m} / \mathrm{s}(\mathrm{PGCDER}, 2007)$ à $1,4 \cdot 10^{-4}$ m/s (PUB, 2011), les valeurs les plus faibles provenant d'Amérique du Nord (CVC, 2010; NCDWQ, 2017; Prince George's County, Maryland, 1999). Ceci peut sembler étonnant, les pluies en Amérique du Nord étant les plus intenses des pays étudiés, à l'exception de Singapour ( $c f$. Tableau I). Nous discuterons de l'implication de ces différences sur la conception des géométries des systèmes en fonction des objectifs fixés aux ouvrages dans les sections qui suivent. 


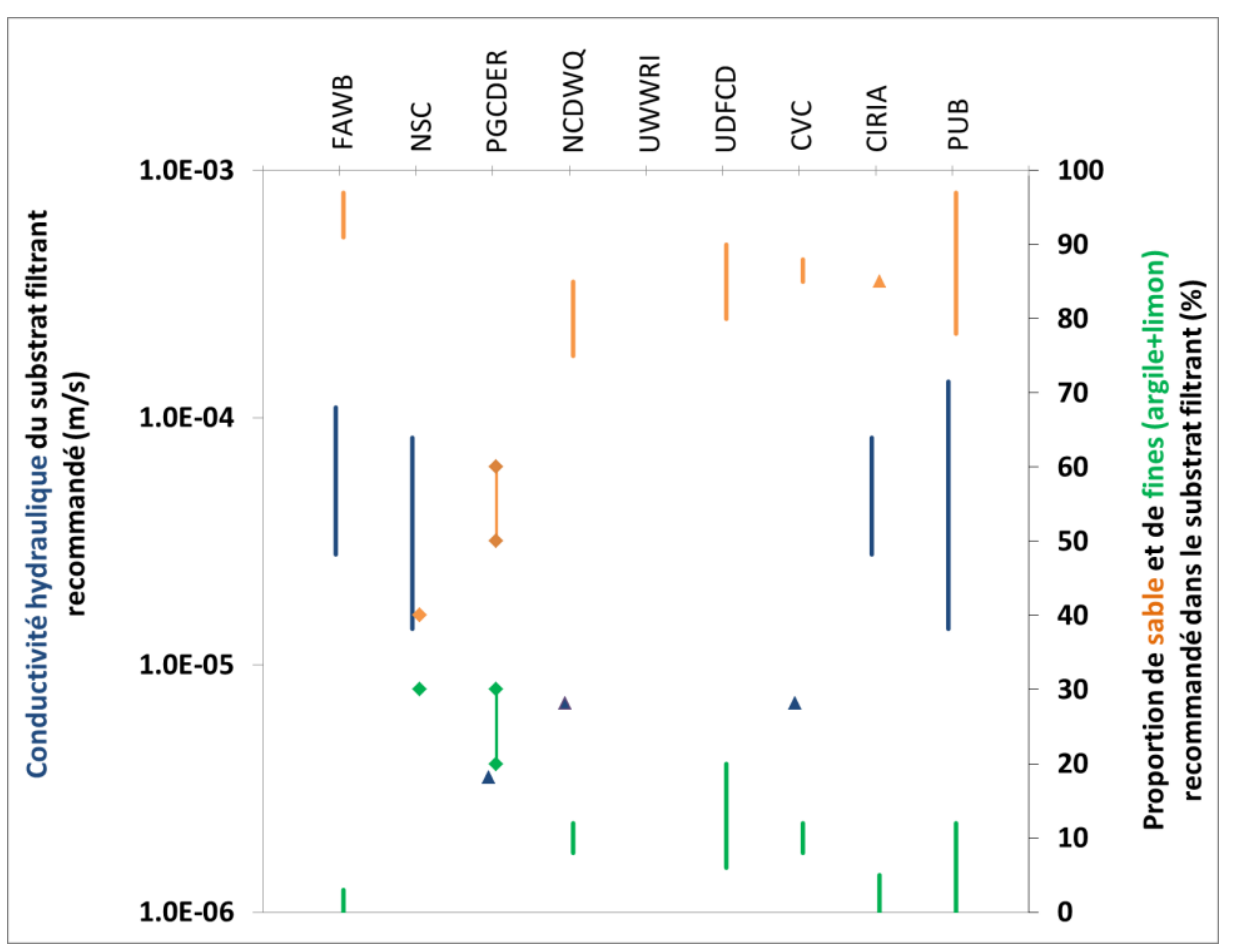

Figure 3 : Nature des substrats de filtration recommandés. Les lignes représentent des gammes de valeurs recommandées, les triangles représentent une valeur minimale, les losanges représentent une recommandation non en termes de granulométrie mais en matériaux constituant le substrat (sable et terre végétale)

La plupart des guides précisent également la granulométrie du substrat filtrant. Relié à la conductivité hydraulique (même si d'autres facteurs, tels que la compaction du sol peuvent aussi influencer $K_{s}$ ), ce paramètre est parfois considéré comme suffisant pour assurer le bon fonctionnement hydraulique du substrat (NCDWQ, 2017; UDFCD, 2015a) et d'autres fois considéré comme un paramètre indicatif à ajuster pour obtenir le $K_{s}$ souhaité (CIRIA, 2015; FAWB, 2009; PUB, 2011). De manière générale, on trouve des recommandations pour une proportion de sable importante $(>80 \%)$ et une teneur faible en argiles et limons (<20\% cumulés), ce qui est présenté en Figure 3 (CIRIA, 2015; CVC, 2010; FAWB, 2009; NCDWQ, 2017; PUB, 2011; UDFCD, 2015a). Deux guides fournissent des recommandations non pas en termes de granulométrie mais de matériaux utilisés pour constituer le substrat : sable, compost et terre végétale, la terre végétale étant elle-même un mélange de sables, limons, argiles granulométriques et matière organique en proportions variables (NSC, 2008; PGCDER, 2007). On note que la proportion de sable recommandée par ces deux guides est relativement faible mais que cette valeur doit être comprise comme une valeur minimale sachant qu'un apport de sable granulométrique sera associé à la terre végétale.

La teneur en matière organique du substrat est également fréquemment citée. La matière organique contribue à la capacité du sol à retenir l'eau, ce qui est favorable pour le développement de la végétation (FAWB, 2009), mais aussi à adsorber les contaminants dissous (LeFevre et al., 2014). Un guide mentionne que la proportion de matière organique recommandée a été abaissée suite à un retour d'expérience démontrant un lessivage de nutriments à partir des matériaux tels que le compost (UDFCD, 2015a). Les valeurs les plus fréquentes sont situées entre 3 et 10\% de matière organique (CIRIA, 2015; CVC, 2010; NCDWQ, 2017; PUB, 2011; UDFCD, 2015), mais deux guides proposent une proportion de compost pouvant atteindre $30 \%$ sans préciser la teneur en matière organique exacte du substrat final (NSC, 2008; PGCDER, 2007).

Le pH du sol peut jouer un rôle par rapport à la spéciation et donc la rétention des métaux dissous (LeFevre et al., 2014). Les ouvrages de biorétention peuvent également servir de tampon de $\mathrm{pH}$ dans le cas où ils reçoivent des eaux de ruissellement acides (Roy-Poirier et al., 2010). On retrouve des recommandations de $\mathrm{pH}$ du substrat à l'eau variant entre 5,2 (NSC, 2008) et 8.5 (CIRIA, 2015), la gamme la plus courante étant entre 5,5 et 7,5 (CVC, 2010; FAWB, 2009; PUB, 2011).

Il est essentiel que le sol possède suffisamment de nutriments pour que la végétation puisse bien se développer - cependant des teneurs en nutriments trop élevées peuvent mener à un lessivage de ces composés dans les eaux traitées. Ainsi, on trouve des limitations des teneurs en azote et en phosphore dans le substrat. Des concentrations maximales en azote de $0,1 \%$ (FAWB, 2009) et de 0,1-0,3\%ù (CIRIA, 2015) ont ainsi été proposées. Des concentrations limites en phosphore sont également proposées mais les valeurs sont difficilement comparables entre les guides, car liées à des formes et des mesures de phosphore différentes. On retrouve ainsi des valeurs limites exprimées en phosphore total (UDFCD, 2015a), en orthophosphate (FAWB, 2009), en $\mathrm{P}_{2} \mathrm{O}_{5}$ (NSC, 2008), en phosphore extractible à l'eau (CIRIA, 2015) et en P-index, un facteur censé estimer le risque de relargage de phosphore vers les eaux de surface (CVC, 2010; NCDWQ, 2017). 
Des niveaux excessifs de sel dans le substrat peuvent gêner le développement des plantes (FAWB, 2009). Ainsi, quatre guides donnent des limites de la conductivité électrique du substrat à ne pas dépasser (CIRIA, 2015; FAWB, 2009; PUB, 2011; UDFCD, 2015a). Ces valeurs varient de 0.63 mS/cm (PUB, 2011) à 6 mS/cm (UDFCD, 2015a).

\subsection{Critères et méthodes de dimensionnement}

Les ouvrages de biorétention sont des systèmes multifonctionnels contribuant aussi bien à la gestion du flux polluant qu'à la gestion des flux hydriques associés aux eaux de ruissellement de voirie. Ainsi, dans les guides on trouve une diversité de critères et règles de dimensionnement pour fixer les paramètres de l'ouvrage déterminant le volume de stockage disponible et le débit de traitement. Ces paramètres de dimensionnement sont : le ratio $(b)$ entre la surface de l'ouvrage $(A)$ et la surface de son bassin versant $(B V)$, la hauteur maximale de stockage d'eau à la surface $\left(h_{\text {eau }}\right)$ et, plus rarement, la conductivité hydraulique $\left(K_{s}\right)$ et l'épaisseur des couches du filtre $\left(P_{\text {filtre }}\right)$.

Dans la section précédente, nous avons vu que la plupart des guides présentent une gamme de valeurs acceptables de $K_{s}$ et de $P_{\text {filtre }}$. De manière similaire, chaque guide propose des limites à $h_{\text {eau }}$ (Tableau III) afin de restreindre le temps de vidange nécessaire (UWWRI, 2006), limiter la période de submersion des plantes (NCDWQ, 2017; UWWRI, 2006), et, cas d'installation dans un lieu public, d'assurer la sécurité des personnes ainsi que l'esthétique et l'intégration de l'ouvrage dans le paysage (UDFCD, 2015a; UWWRI, 2006). Le ratio $b$ est généralement issu de la méthode de dimensionnement, dont les différents types sont décrits ci-dessous. On observe que ce ratio est très variable, entre 2\% (CIRIA, 2015; FAWB, 2009; UDFCD, 2015a,) et 20\% (CVC, 2010). Ces différences sont liées à la diversité des régimes climatiques (par exemple, les guides préconisant la plus petite surface de l'ouvrage sont ceux provenant des régions les moins pluvieuses), mais aussi de la variabilité en termes de critères et de méthodes de dimensionnement.

\begin{tabular}{|c|c|c|c|c|}
\hline Guide & $h_{\text {pluie }}$ à traiter & $t_{\text {vidange }}$ & $h_{\text {eau }}$ & $b$ \\
\hline 1 & ex. $10-15 \mathrm{~mm}$ & $24-48 \mathrm{~h}$ & $20-50 \mathrm{~cm}$ & $\sim 2 \%$ \\
\hline 2 & $27 \mathrm{~mm}$ & $8 \mathrm{~h}$ & $20-30 \mathrm{~cm}$ & $5-8 \%^{\circ}$ \\
\hline 3 & $23-25 \mathrm{~mm}$ & $48 \mathrm{~h}$ & $<30 \mathrm{~cm},<60 \mathrm{~cm}^{+}$ & Ex. $7.2 \%^{*}$ \\
\hline 4 & $25 \mathrm{~mm}$ & $12 \mathrm{~h}$ & $<30 \mathrm{~cm}$ & $8-11 \%$ \\
\hline 5 & - & $24 \mathrm{~h}$ & $<46 \mathrm{~cm}$ & Ex. $15 \%$ \\
\hline 6 & $15 \mathrm{~mm}$ & $12 \mathrm{~h}$ & $<30 \mathrm{~cm}$ & $>2 \%^{\circ}$ \\
\hline 7 & - & $24 \mathrm{~h}$ & $15-25 \mathrm{~cm}$ & $7-20 \%$ \\
\hline 8 & - & $24-48 \mathrm{~h}$ & $15-30 \mathrm{~cm}$ & $2-4 \%$ \\
\hline 9 & - & - & $<30 \mathrm{~cm}$ & Ex. $5 \%$ \\
\hline
\end{tabular}

Tableau III : Valeurs recommandées pour le dimensionnement des ouvrages de biorétention ( $h_{\text {pluie }}$ est la hauteur de pluie à traiter, $t_{\text {vidange }}$ est le temps de vidange, $\boldsymbol{h}_{\text {eаu }}$ est la profondeur du stockage à la surface et $\boldsymbol{b}$ est le ratio de la surface de l'ouvrage à celle du bassin versant d'apport). ${ }^{1}(\mathrm{FAWB}, 2009)$, ${ }^{2}$ (NSC, 2008), ${ }^{3}$ (PGCDER, 2007), ${ }^{4}$ (NCDWQ, 2017), ${ }^{5}$ (UWWRI, 2006), ${ }^{6}$ (UDFCD, 2015a), ${ }^{7}$ (CVC, 2010), ${ }^{8}$ (CIRIA, 2015), ${ }^{9}\left(\right.$ PUB, 2011). ${ }^{+}$En cas d'un objectif supplémentaire de détention, ${ }^{\circ}$ Ratio de la surface de l'ouvrage à la surface imperméable du bassin versant, *Ex. signifie que la valeur est un exemple donnée et non une valeur recommandée.

\section{Dimensionnement empirique}

Le guide néo-zélandais présente une méthode de conception complètement empirique : la surface de l'ouvrage est fixée à un pourcentage ( 5 ou $8 \%$, selon la présence ou non d'un dispositif de traitement en aval) de la surface imperméable du bassin versant, avec une hauteur de stockage à la surface de $20-30 \mathrm{~cm}$. Ces dimensions visent à traiter le premier flot, défini comme un tiers de l'événement biannuel de durée 24h, de lame d'eau égale à 26.6 mm (NSC, 2008).

\section{Dimensionnement par volume de stockage}

La méthode de dimensionnement la plus fréquente se base sur le volume d'eau pouvant être stocké par l'ouvrage (Figure 4). Selon cette méthode, le concepteur doit calculer le volume à traiter, puis dimensionner l'ouvrage de telle façon que ce volume puisse être stocké dans l'ouvrage. Les compartiments de l'ouvrage considérés comme contribuant au stockage diffèrent entre les guides : deux guides ne comptabilisent que le volume de stockage disponible à la surface (CIRIA, 2015; NCDWQ, 2017), un guide propose le stockage de 75\% du volume calculé à la surface de l'ouvrage y compris dans les ouvrages de prétraitement avec les dispositifs de prétraitement (PGCDER, 2007), un guide compte le stockage à la surface et une partie de la porosité du filtre (UDFCD, 2015a), alors qu'un dernier ne prend en compte que le volume de stockage disponible dans la porosité du filtre $(\mathrm{CVC}, 2010)$. Le concepteur doit choisir $h_{\text {eau }}$ et/ou $P_{\text {filtre }}$ généralement dans une gamme préconisée, afin de pouvoir calculer la surface de l'ouvrage nécessaire. Selon cette approche, le concepteur peut limiter la surface de l'ouvrage nécessaire en sélectionnant la $h_{\text {eau }}$ et/ou la $P_{\text {filtre }}$ maximale acceptable.

Le volume à traiter est associé à une hauteur de précipitation définie selon le cas comme un petit événement pluvial fréquent (FAWB, 2009), le premier flot (NCDWQ, 2017), le water quality volume ou WQV (CVC, 2010; PGCDER, 2007; UDFCD, 2015a) ou l'événement pluvial annuel de durée critique (CIRIA, 2015). La définition exacte du petit événement fréquent varie selon le territoire mais un exemple donné est l'événement de période de retour de trois mois (FAWB, 2009). Le premier flot est défini par NCDWQ (2017) comme le volume généré par les premiers 25 mm de pluie sans définition probabiliste. Le $W Q V$ correspond au $80^{\mathrm{eme}}$ percentile des événements pluvieux produisant du ruissellement (UDFCD, 2015b). Les hauteurs d'eau correspondant à ces critères sont parfois citées dans les guides (Tableau III) ; d'autres fois, seule 
la méthode de calcul de la hauteur d'eau est précisée (CIRIA, 2015; CVC, 2010). Parfois, d'autres critères hydrologiques, auxquels on associe des volumes plus contraignants, sont également présentés, tels que l'écrêtement du débit de pointe (CIRIA, 2015; UDFCD, 2015a), la détention d'un volume d'eau (par exemple, la pluie annuelle de 24h) pendant plusieurs jours pour la protection des berges (NCDWQ, 2017; PGCDER, 2007), le maintien des volumes de ruissellement sortant d'un bassin versant au niveau pré-développement jusqu'à un événement pluvial précisé (CIRIA, 2015; FAWB, 2009; PGCDER, 2007) ou l'infiltration d'un volume d'eau afin de favoriser la recharge de la nappe phréatique (calculé par une équation empirique en fonction du type de sol, PGCDER, 2007).

Deux guides couplent le dimensionnement par volume de stockage avec une contrainte associée au temps de traitement (cf. lignes pointillées en Figure 4). Ces guides recommandent de vérifier que le temps de vidange nécessaire est inférieur à une valeur recommandée. Puisque, selon les recommandations de drainage de ces guides, le débit est limité par la conductivité hydraulique de la couche la moins perméable du sol (cf. § 3.2), dans ces guides, le temps de vidange est calculé par la loi de Darcy en considérant l'ouvrage rempli au niveau $h_{\text {eau }}(E q .5$ en Figure 4). Si le temps de vidange calculé est trop long, il sera nécessaire d'augmenter $K_{s}$ ou bien de diminuer $h_{\text {eau }}$ en augmentant $A$ par conséquent (CIRIA, 2015; PGCDER, 2007). Bien que les autres guides n'introduisent pas ce critère explicitement, le temps de vidange est implicitement limité par les contraintes de $K_{s}$ et de $h_{\text {eau }}$ imposés ; de plus, ils citent souvent une gamme de temps de vidange recommandée (Tableau III).
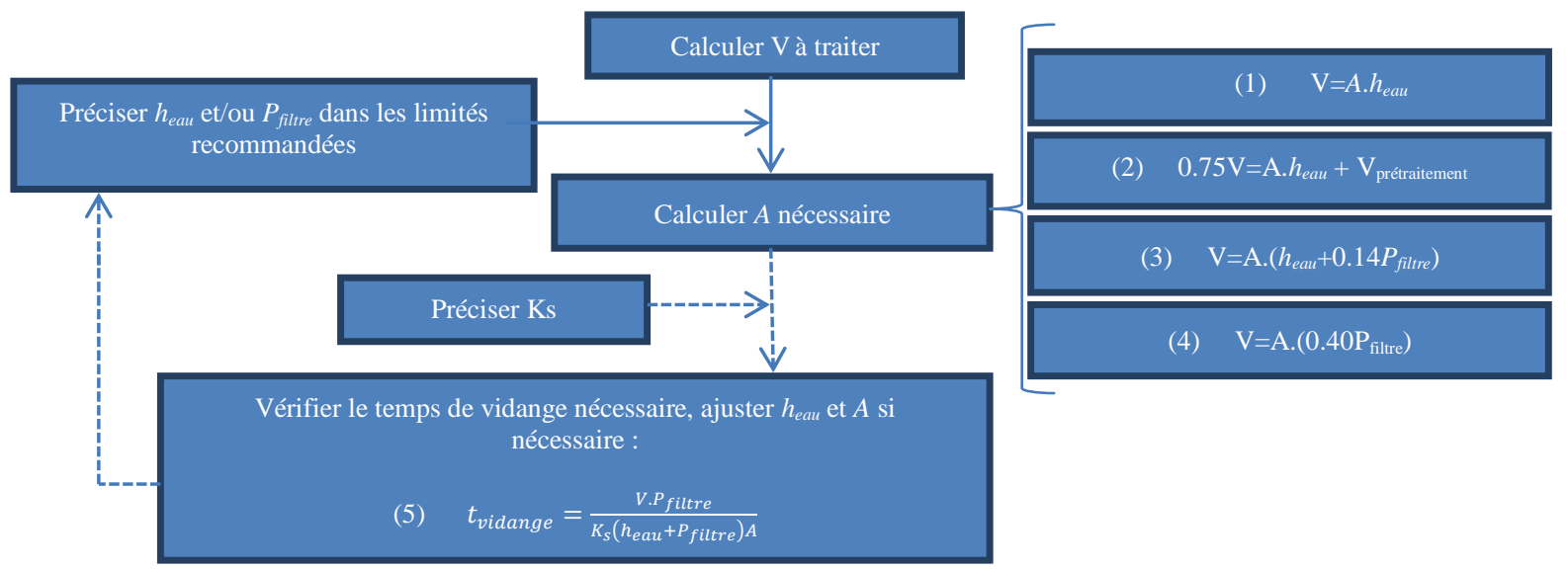

Figure 4 : Schéma de la procédure de dimensionnement par volume de stockage avec les équations employés par guide : ${ }^{1}$ (CIRIA, 2015; NCDWQ, 2017), ${ }^{2}$ (PGCDER, 2007), ${ }^{3}$ (UDFCD, 2015a), ${ }^{4}$ (CVC, 2010), ${ }^{5}$ (CIRIA, 2015; PGCDER, 2007).

Ces approches, basées sur des calculs simples, ont l'avantage d'être relativement faciles à appliquer et d'avoir une justification physique. Cependant, la variabilité des approches montre qu'il n'y a pas consensus sur les objectifs d'interception volumique, ni sur la méthode de déclinaison de ces objectifs en hauteur d'eau à traiter, ni sur les compartiments de l'ouvrage à prendre en compte dans le calcul du volume de stockage. Ce choix se base donc sur le jugement de l'auteur du guide. De plus, cette approche repose sur la notion d'un événement pluvial isolé où l'ouvrage est vide au début et doit se vider à la fin. Elle ne prend pas en compte la dynamique de remplissage et de vidange de l'ouvrage qui dépend de l'enchaînement des pluies et donc du climat. La prise en compte du temps de vidange vise à assurer la vidange du système entre deux événements pluvieux, mais la valeur de ce paramètre n'est pas corrélée avec les caractéristiques hydroclimatiques des pays, ce qui indique qu'elle reflète plus le jugement des auteurs du guide qu'une réalité physique.

\section{Méthodes basées sur un objectif de performance}

Une troisième approche de dimensionnement se base sur l'estimation de la performance épuratoire ou hydraulique de l'ouvrage pour une configuration spécifiée. Ce type d'approche, qu'on retrouve en Australie, à Singapour et au Wisconsin, s'appuie sur des abaques, outil simple mais rigide, ou sur un outil de modélisation, plus flexible mais plus compliqué à utiliser. A Singapour, le critère de dimensionnement est toujours une réduction du flux polluant. Dans le guide australien, comme dans le guide du Wisconsin, plusieurs critères possibles sont proposés parmi lesquels l'utilisateur doit choisir en fonction du règlement local ou des objectifs du projet (FAWB, 2009; UWWRI, 2006).

En Australie et à Singapour, il existe des abaques pour les climats locaux reliant la performance attendue en termes de rétention du flux polluant à la valeur de $b$ pour un ouvrage ayant une conductivité hydraulique et une $h_{\text {eau }}$ fixées. Ces courbes, qui prennent en compte les matières en suspension, l'azote total et le phosphore, sont elles-mêmes issues de simulations de longue durée avec le modèle MUSIC qui couple un modèle hydrologique avec un modèle simple de qualité prenant en compte la réduction des concentrations de ces polluants. La procédure de dimensionnement utilisant ces abaques est illustrée en Figure 5 ; on note que les critères de performance ainsi que les configurations de l'ouvrage considérés sont limités à ceux pris en compte par cet outil. Pour un ouvrage de configuration moins courante ou pour d'autres objectifs (par 
exemple, la limitation du ruissellement au niveau avant l'urbanisation), le concepteur peut effectuer lui-même une simulation de longue durée avec l'outil MUSIC (FAWB, 2009; PUB, 2011). Dans ce cas, l'utilisateur doit évaluer, de façon itérative, les paramètres de l'ouvrage afin d'atteindre une configuration avec le niveau de performance souhaitée (Figure 6).

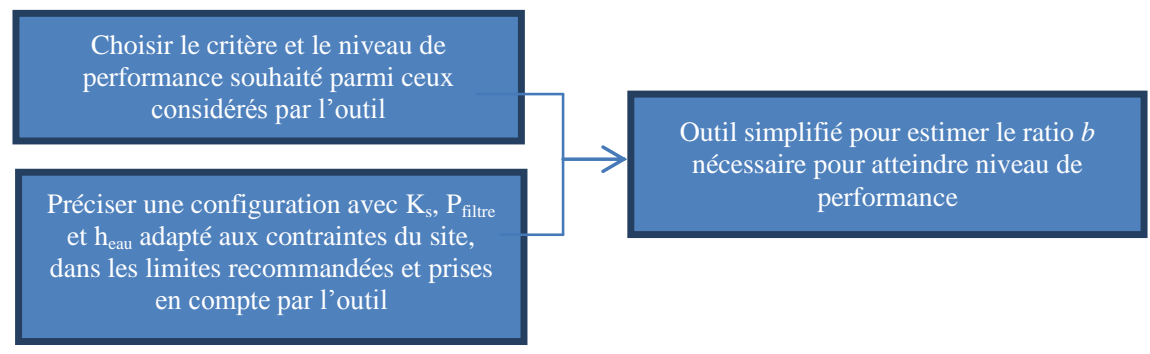

Figure 5 : Schéma de la procédure de dimensionnement par un outil simplifié pour estimer le ratio $b$ nécessaire pour atteindre une performance souhaitée (FAWB, 2009; PUB, 2011; UWWRI, 2006)

$\mathrm{Au}$ Wisconsin, le dimensionnement se base uniquement sur un modèle hydrologique, appelé RECARGA. Ce modèle peut retourner le ratio $b$ nécessaire pour atteindre un objectif de rétention d'une proportion du volume de ruissellement pour une configuration fixée. La procédure de dimensionnement par cet outil est également représentée par la Figure 5 ; on note que cet outil permet plus de flexibilité en termes de configurations de l'ouvrage pouvant être prises en compte que les abaques, mais qu'il reste rigide en termes d'objectif à atteindre. Si le concepteur souhaite considérer d'autres critères de dimensionnement (tel qu'un objectif de recharge de la nappe ou d'écrêtement de débit) ou comparer la performance de différentes configurations possibles, le modèle est aussi capable de retourner un bilan hydrique pour une chronique de pluie et ruissellement et une configuration d'ouvrage spécifié. Ce bilan prend en compte les volumes générés par le bassin versant, la surverse de l'ouvrage, la recharge de la nappe phréatique, l'évaporation, l'écoulement dans le drain, ainsi que la teneur en eau du substrat. Un utilisateur pourra ainsi dimensionner un ouvrage, en se servant de ce bilan pour évaluer différents critères de performance, et en faisant des itérations afin de trouver une configuration optimale, comme avec MUSIC (Figure 6). (UWWRI, 2006).

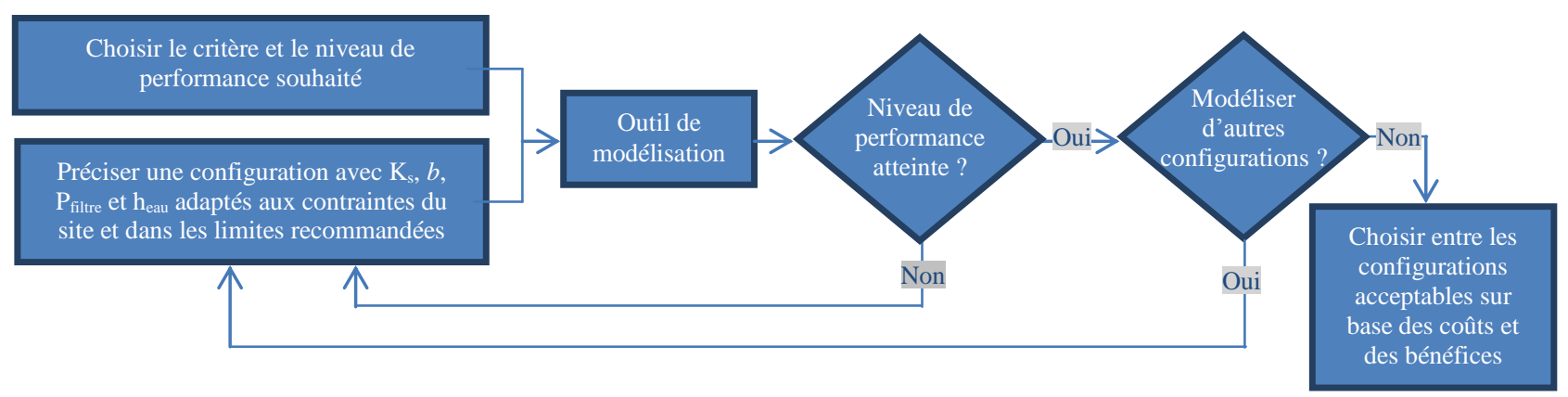

Figure 6 : Schéma de la procédure de dimensionnement par modélisation de performance libre (FAWB, 2009; PUB, 2011; UWWRI, 2006)

Dans le cas du dimensionnement par une modélisation de performance libre (MUSIC ou la deuxième utilisation de RECARGA, Figure 6), on peut généralement trouver plusieurs configurations pouvant atteindre la performance souhaitée. Il peut donc être intéressant que le concepteur étudie plusieurs configurations possibles pour le site étudié afin de choisir la plus avantageuse et/ou la moins onéreuse. La liberté laissée au concepteur pour adapter l'ouvrage aux contraintes particulières du site est un avantage de cette méthode mais est l'aboutissement d'une procédure de conception relativement complexe. On souligne aussi que ces modèles peuvent impliquer des incertitudes importantes, associées à l'estimation des paramètres ou à la description simplifiée des processus, qui peuvent ne pas toujours être bien appréciées par les utilisateurs.

\subsection{Critères de choix des végétaux}

Les végétaux sont un composant essentiel d'un système de biorétention, le distinguant d'un système de filtration simple. Le rôle des végétaux est discuté dans la majorité des guides étudiés. On peut ainsi distinguer les rôles physiques, hydrologiques et épuratoires. Deux rôles principaux des plantes pour le bon fonctionnement de ces systèmes sont ceux associés à l'interaction physique des racines avec le substrat: la limitation du colmatage du substrat en renouvelant sa porosité (CIRIA, 2015; FAWB, 2009; NCDWQ, 2017; PUB, 2011; UWWRI, 2006) et la stabilisation du substrat, ce qui permet de minimiser l'érosion (CIRIA, 2015; PUB, 2011). Hydrologiquement, les végétaux contribuent à la réduction des volumes ruisselés par évapotranspiration (PGCDER, 2007). Enfin, on attend que les plantes, surtout la partie racinaire, contribuent à abattre la pollution véhiculée par les eaux de ruissellement (NCDWQ, 2017; UWWRI, 2006). Plus spécifiquement, elles supportent une communauté microbienne capable de dégrader certains polluants (PUB, 2011), elles apportent de l'oxygène au milieu (PUB, 2011), et peuvent exporter certains polluants du système (CIRIA, 2015; NCDWQ, 2017; PGCDER, 2007), notamment les nutriments (FAWB, 2009; PGCDER, 2007). 
Chaque guide propose des conseils de choix des espèces végétales à planter dans les ouvrages de biorétention. Etant donné les conditions difficiles au sein d'un système de biorétention, il est souvent préconisé de semer une grande variété d'espèces afin de permettre une autosélection des espèces les plus adaptées (CIRIA, 2015; FAWB, 2009; NCDWQ, 2017; PUB, 2011; UWWRI, 2006) et les critères de choix les plus souvent cités sont ceux liés à la survie des plantes, notamment la tolérance à la pollution apportée par le ruissellement (CVC, 2010; PGCDER, 2007; PUB, 2011), aux caractéristiques du substrat filtrant (CIRIA, 2015; FAWB, 2009; PGCDER, 2007; PUB, 2011; UDFCD, 2015a; UWWRI, 2006) et aux conditions hydrologiques variables (CIRIA, 2015; CVC, 2010; FAWB, 2009; NCDWQ, 2017; NSC, 2008; PGCDER, 2007; PUB, 2011; UDFCD, 2015a; UWWRI, 2006). En effet, la conception hydrologique des ouvrages de biorétention implique que le substrat filtrant sera parfois inondé et parfois très sec, ce qui doit impérativement être pris en compte dans le choix des espèces. Plusieurs guides reconnaissent également que les conditions hydrologiques peuvent varier au sein de l'ouvrage et recommandent l'utilisation d'espèces différentes, plus ou moins adaptées à des conditions humides, en fonction de la situation au sein de l'ouvrage (CIRIA, 2015; CVC, 2010; FAWB, 2009; NCDWQ, 2017; PGCDER, 2007; PUB, 2011).

Certains guides proposent que les ouvrages de biorétention contribuent à la biodiversité en milieu urbain (CIRIA, 2015; FAWB, 2009; PUB, 2011) et le guide du Royaume Uni considère même la biodiversité comme un des objectifs principaux des techniques alternatives pour la gestion des eaux pluviales (CIRIA, 2015). L'utilisation d'espèces autochtones est aussi recommandée (CIRIA, 2015; CVC, 2010; NSC, 2008; PGCDER, 2007).

Il est également couramment suggéré de choisir des végétaux ayant un système racinaire extensif afin de favoriser les processus de phytorémédiation (CIRIA, 2015; FAWB, 2009; NSC, 2008; PGCDER, 2007; PUB, 2011). Deux guides présentent une liste d'espèces particulièrement efficaces pour la rétention de nutriments, établie sur la base de tests en colonne (FAWB, 2009; PUB, 2011). Cependant, un critère qui brille par son absence dans les guides est la capacité des plantes à accumuler les polluants. En effet, la phytoextraction est un processus d'importance mineure pour le devenir des polluants dans ces types d'ouvrages (Leroy, 2015) et d'autres paramètres s'avèrent plus efficaces pour optimiser la dépollution.

Certains guides proposent des critères de choix des plantes visant à contrôler les risques pouvant être posés par les ouvrages de biorétention. Par exemple, il est conseillé d'éviter les plantes avec racines pivotantes qui peuvent endommager les systèmes de drainage et d'étanchéification (UDFCD, 2015a) ou créer des écoulements préférentiels dans le sol (PUB, 2011). Par ailleurs, il est recommandé à veiller à ce que les végétaux n'engendrent pas de risque vis-à-vis des infrastructures à proximité de l'ouvrage et que la visibilité de sécurité soit maintenue en cas d'installation à proximité d'une route (NCDWQ, 2017; NSC, 2008).

Comme ce type d'ouvrage doit s'intégrer dans le paysage urbain, plusieurs guides préconisent la prise en compte de l'esthétique, et l'intégration dans le paysage, dans le choix des plantes (CIRIA, 2015; NCDWQ, 2017; UDFCD, 2015a; UWWRI, 2006). Le guide du Royaume Uni affirme que le choix des végétaux dépend fortement du site et nécessite l'avis d'un paysagiste professionnel (CIRIA, 2015).

Il est généralement considéré souhaitable de planter les végétaux à une densité importante afin d'augmenter la densité racinaire, maintenir la porosité du sol à la surface, promouvoir une distribution homogène de l'écoulement, augmenter l'évapotranspiration et réduire la présence de mauvaises herbes (FAWB, 2009). Les densités recommandées varient en fonction des types de plantes et se trouvent dans la gamme de 1 à10 plantes $/ \mathrm{m}^{2}$ (CIRIA, 2015; FAWB, 2009; NSC, 2008; PUB, 2011).

La majorité des guides comprend une liste d'espèces recommandées qui peuvent aller de plantes couvre-sol ou herbacées à des espèces ornementales vivaces jusqu'aux arbustes ou arbres (CVC, 2010; FAWB, 2009; NCDWQ, 2017; NSC, 2008; PGCDER, 2007; PUB, 2011; UDFCD, 2015a). On ne discutera pas en détail les espèces particulières qui varient en fonction de la région géographique concernée comme on le voit dans les Figures $7(a)$ et $7(b)$ qui montrent, respectivement, des ouvrages de biorétention avec une végétation typique en Amérique du Nord, en Caroline du Nord et en AustralieOccidentale. 

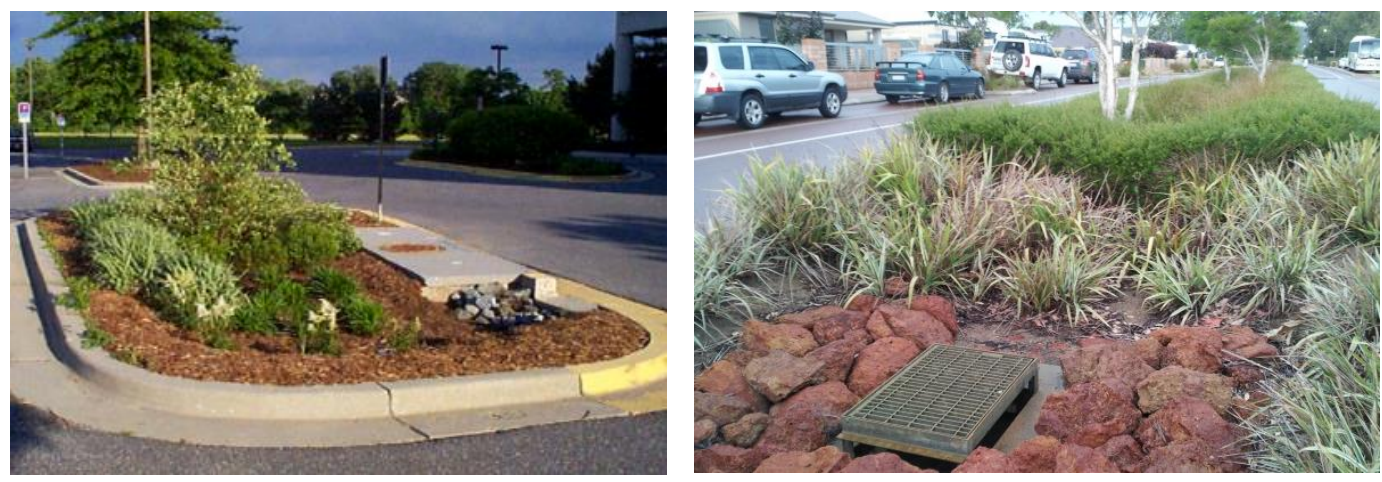

Figure 7 : Exemple d'un ouvrage typique (a) de biorétention aux Etats-Unis (NCDWQ, avec permission) et (b) de biofiltration en Australie (Emily Payne, Monash University, avec permission)

\subsection{Entretien et maintenance}

L'entretien des ouvrages de biorétention est essentiel pour leur pérennité. Une majorité des guides étudiés souligne l'importance d'établir un plan d'entretien régulier dès la mise en place du système (CIRIA, 2015; CVC, 2010; FAWB, 2009; NSC, 2008; PGCDER, 2007). Deux guides n'évoquent pas du tout l'entretien (UDFCD, 2015a; UWWRI, 2006) alors que deux autres apportent des préconisations d'entretien sans parler de la nécessité de mettre en place un plan d'entretien régulier (NCDWQ, 2017; PUB, 2011). On peut distinguer les opérations d'entretien courant et la maintenance plus lourde à effectuer à une fréquence plus faible.

L'entretien régulier inclut, d'abord, des opérations d'entretien spécifiques aux espaces verts en milieu urbain : l'enlèvement des mauvaises herbes (CVC, 2010; FAWB, 2009; NCDWQ, 2017; NSC, 2008; PGCDER, 2007; PUB, 2011), l'enlèvement et le remplacement des plantes mortes (CIRIA, 2015; CVC, 2010; FAWB, 2009; NCDWQ, 2017; NSC, 2008; PGCDER, 2007; PUB, 2011), la taille des plantes (CVC, 2010; FAWB, 2009; NCDWQ, 2017; NSC, 2008; PGCDER, 2007) et l'ajout de paillage (CVC, 2010; NCDWQ, 2017; NSC, 2008; PGCDER, 2007). Contrairement aux espaces verts, l'irrigation et l'apport d'engrais n'ont pas lieu d'être, l'alimentation de l'ouvrage s'en chargeant, surtout si l'ouvrage de biorétention possède une zone de stockage au fond (FAWB, 2009; PUB, 2011). Cependant, deux guides mentionnent que l'irrigation peut être nécessaire pendant les périodes sèches (NSC, 2008; UDFCD, 2015a). Le guide du Colorado, la plus aride des régions étudiées, préconise en particulier de choisir des plantes adaptées à la sécheresse et nécessitant peu d'eau (UDFCD, 2015a). D'autres guides affichent un besoin d'irrigation mais uniquement lors de la phase d'installation des plantes (FAWB, 2009; NCDWQ, 2017; PGCDER, 2007). Seul le guide de Singapour précise que la fertilisation peut être nécessaire si l'état des plantes le justifie (PUB, 2011).

D'autres opérations recommandées selon une fréquence importante (tous les 1 à 12 mois) sont l'enlèvement de déchets (CIRIA, 2015; CVC, 2010; FAWB, 2009; NSC, 2008; PGCDER, 2007; PUB, 2011), l'inspection visuelle des dispositifs d'arrivée et de prétraitement (CIRIA, 2015; CVC, 2010; FAWB, 2009; NSC, 2008; PUB, 2011), de surverse (CIRIA, 2015; FAWB, 2009; NSC, 2008) et du substrat filtrant (CIRIA, 2015; CVC, 2010; FAWB, 2009; NCDWQ, 2017; NSC, 2008; PGCDER, 2007). Les résultats de ces inspections, peuvent entrainer d'autres interventions, telles que l'enlèvement des sédiments (CIRIA, 2015; CVC, 2010; FAWB, 2009; NCDWQ, 2017; NSC, 2008; PUB, 2011) ou la réparation des zones de substrat érodé (CIRIA, 2015; CVC, 2010; FAWB, 2009; NCDWQ, 2017; PGCDER, 2007). L'inspection du système de drainage est fréquemment recommandée et peut se faire en même temps (CIRIA, 2015; CVC, 2010) ou à un pas moins régulier (FAWB, 2009; NCDWQ, 2017; NSC, 2008).

Afin de lutter contre le colmatage, parmi les guides recommandant le paillage, trois proposent l'enlèvement du paillage et des sédiments à sa surface à une fréquence annuelle à trisannuelle (CIRIA, 2015; NCDWQ, 2017; PGCDER, 2007). Lorsque la vidange de l'ouvrage ralentit excessivement, des interventions de maintenances plus lourdes peuvent être déclenchées telles que le remplacement de la première couche de substrat filtrant (CIRIA, 2015; NSC, 2008) ou de la couche de transition (NSC, 2008). Différents guides estiment la durée de vie totale de l'ouvrage avant que toute l'épaisseur de la couche de substrat filtrant ne soit remplacée. Ces estimations varient de 10-15 ans (FAWB, 2009) à 20 ans (NCDWQ, 2017) ou plus (UWWRI, 2006).

On souligne que l'existence de recommandations de maintenance ne suffit pas pour garantir leur bonne prise en compte. En effet, des études menées aux Etats-Unis, en Ecosse et en Australie montrent que l'entretien des techniques est généralement insuffisant, malgré l'existence de recommandations dans les guides, ce qui nuit au bon fonctionnement à long terme des ouvrages (Flynn et al., 2012; Schlüter and Jefferies, 2005; Thomas et al., 2016).

\section{Conclusions}

Dans cet article, nous avons exploré les recommandations issues de guides publiés à l'international pour la conception et la maintenance des systèmes décentralisés de stockage et filtration naturelle des eaux de ruissellement, dits ouvrages de 
biorétention ou biofiltration. Ces guides constituent une source riche d'informations issues des retours d'expérience dans les pays anglophones où ces techniques sont pratiquées depuis plusieurs années, suivant un mouvement initié à Maryland aux Etats-Unis en 1993.

A l'heure actuelle, en France, la conception des ouvrages végétalisés de stockage et dépollution à la source des eaux de ruissellement urbaines n'est pas encadrée par un guide opérationnel avec un niveau de détail comparable à ceux étudiés et est, de ce fait, réalisée selon les méthodes, généralement empiriques, propres à chaque aménageur ou bureau d'études. L'établissement d'un guide de conception en France paraitrait un bon moyen d'établir un vocabulaire commun entre acteurs opérationnels, de mieux cadrer les objectifs attendus et d'améliorer le partage des connaissances. Les guides anglophones peuvent servir d'exemple mais il est nécessaire de considérer la pertinence de leur structure et leur contenu au contexte français.

Les guides étudiés cadrent fortement de nombreux éléments de conception de ces systèmes (dispositifs de drainage et de surverse, composition et épaisseur du substrat, types de plantes...), ce qui a tendance à limiter la variabilité des pratiques opérationnelles. Un avantage de cette standardisation est la possibilité de mieux prévoir, voire de garantir, la performance des ouvrages à partir des études et retours d'expérience sur des dispositifs similaires. Cependant, elle n'est pas sans coût (par exemple, les coûts financiers et environnementaux des travaux pour remplacer le sol naturel par un substrat filtrant spécifique). De plus, on peut s'interroger sur l'utilité d'assurer un même niveau de performance sur tous les ouvrages sachant que l'enjeu associé à la gestion de l'eau et/ou du flux polluant associé est variable selon les sites. Par exemple, on pourrait imaginer qu'une solution plus rustique puisse être appliquée dans une zone résidentielle où l'eau de ruissellement est peu polluée. Une trop grande standardisation dans la conception des ouvrages risque par ailleurs de limiter la créativité des aménageurs et de restreindre les possibilités d'intégration de l'eau dans l'urbanisme et la plurifonctionnalité des usages.

Ces recommandations ont également pour objectif de diffuser les connaissances disponibles en vue d'un fonctionnement optimal des systèmes de biorétention. Toutefois, la capacité d'un guide d'assurer réellement une conception optimale est limitée par l'état des connaissances actuelles sur l'effet de différents paramètres de conception sur la rétention d'eau et des polluants dans ces systèmes complexes. Un guide idéal se baserait sur le plus d'informations fiables disponibles, mais devrait également s'accompagner d'un modèle numérique d'aide à la conception permettant de diversifier les configurations.

Les retours d'expérience à l'international, à travers les guides, constituent une source d'information importante, mais non entièrement transposable du fait de différences de contextes hydroclimatiques, environnementaux, urbains et politiques. De fait, certains objectifs cités dans les guides, tels que la réutilisation des eaux ou le traitement des nutriments, diffèrent des préoccupations françaises. De plus, les problèmes et les contraintes rencontrés (par exemple, différences saisonnières de fonctionnement) dépendent non seulement de la composition et du dimensionnement de l'ouvrage mais du contexte dans lequel il s'inscrit. Ainsi, un guide français couplerait idéalement le retour d'expérience international avec un retour d'expérience local. Ce retour d'expérience devrait inclure des expérimentations in situ, permettant d'évaluer l'efficacité des systèmes à atteindre les objectifs propres au contexte français (tel que la rétention de micropolluants) et pourrait également inclure des expériences en conditions contrôlées permettant d'identifier l'effet de différents paramètres sur la performance.

\section{Remerciements}

Ce travail a été conduit dans le cadre de l'Observatoire de Polluants Urbains de la région Parisienne (OPUR) et le projet de recherche ROULEPUR. Les auteurs remercient vivement les partenaires d'OPUR (AESN, SIAAP, CD92, CD93, CD94, Ville de Paris), l'ONEMA et l'Agence de l'Eau Seine-Normandie pour leur soutien financier.

\section{Bibliographie}

Australian Government, B. of M., 2016. Intensity-Frequency-Duration: Design Rainfalls [WWW Document]. URL http://www.bom.gov.au/water/designRainfalls/ifd/ (accessed 10.18.16).

Canty, J., Frischling, B., David, F., 2016. Weatherbase [WWW Document]. URL http://www.weatherbase.com/ (accessed 2.12.16).

Centre for Ecology and Hydrology, 2016. Flood Estimation Handbook Web Service [WWW Document]. URL https://fehweb.ceh.ac.uk/ (accessed 12.5.16).

CIRIA, 2015. The SuDS Manual (C753). London.

Clar, M.L., Barfield, B.J., O'Connor, T.P., 2004. Stormwater best management practice design guide volume 2 vegetative biofilters.

Communauté de Lyon Direction de l'eau, 2012. Les références pluviométriques du Grand Lyon [WWW Document]. URL http://www.grandlyon.com/fileadmin/user_upload/media/pdf/eau/bilanspluviometriques/201202_gl_coefficientsmontana.pdf (accessed 2.12.16).

CVC (Conservation Valley Conservation), Dhalla, S., Zimmer, C., 2010. Low Impact Development Stormwater Management Planning and Design Guide.

Davis, A.P., 2008. Field Performance of Bioretention: Hydrology Impacts. Journal of Hydrologic Engineering 13, 90-95. doi:10.1061/(ASCE)10840699(2008)13:2(90)

Davis, A.P., 2007. Field Performance of Bioretention: Water Quality. Environmental Engineering Science 24, 1048-1064.

Davis, B., Birch, G., 2010. Comparison of heavy metal loads in stormwater runoff from major and minor urban roads using pollutant yield rating curves. Environmental Pollution 158, 2541-2545. doi:10.1016/j.envpol.2010.05.021

DiBlasi, C.J., Li, H., Davis, A.P., Ghosh, U., 2009. Removal and Fate of Polycyclic Aromatic Hydrocarbon Pollutants in an Urban Stormwater Bioretention Facility. Environmental Science \& Technology 43, 494-502. doi:10.1021/es802090g

Dietz, M.E., Clausen, J.C., 2005. A field evaluation of rain garden flow and pollutant treatment. Water, Air, and Soil Pollution 167, $123-138$.

Ecopluies, 2009. L'infiltration en questions: Recommandations pour la faisabilité, la conception et la gestion des ouvrages d'infiltration des eaux pluviales en milieu urbain. 
Egis Eau, 2011. Zonage Pluvial de Carry le Rouet.

Eriksson, E., Baun, A., Scholes, L., Ledin, A., Ahlman, S., Revitt, M., Noutsopoulos, C., Mikkelsen, P.S., 2007. Selected stormwater priority pollutants - a European perspective. Science of The Total Environment 383, 41-51. doi:10.1016/j.scitotenv.2007.05.028

European Commission (Ed.), 2000. Directive 2000/60/EC for the European Parliament of the Council establishing a framework for the Community action in the field of water policy.

FAWB (Facility for Advancing Water Biofiltration), Hatt, B., Morison, P., Fletcher, T., Deletic, A., 2009. Stormwater Biofiltration Systems: Adoption Guidelines.

Fletcher, T.D., Shuster, W., Hunt, W.F., Ashley, R., Butler, D., Arthur, S., Trowsdale, S., Barraud, S., Semadeni-Davies, A., Bertrand-Krajewski, J.-L., Mikkelsen, P.S., Rivard, G., Uhl, M., Dagenais, D., Viklander, M., 2014. SUDS, LID, BMPs, WSUD and more - The evolution and application of terminology surrounding urban drainage. Urban Water Journal 12, 525-542.

Flynn, K.M., Linkous, B.W., Buechter, M.T., 2012. Operation and Maintenance Assessment for Structural Stormwater BMPs. American Society of Civil Engineers, pp. 3662-3673. doi:10.1061/9780784412312.368

Gasperi, J., Sebastian, C., Ruban, V., Delamain, M., Percot, S., Wiest, L., Mirande, C., Caupos, E., Demare, D., Diallo Kessoo Kessoo, M., Saad, M., Schwartz, J.J., Dubois, P., Fratta, C., Wolff, H., Moilleron, R., Chebbo, G., Cren, C., Millet, M., Barraud, S., C Gromaire, M., 2014. Micropollutants in urban stormwater: occurrence, concentrations, and atmospheric contributions for a wide range of contaminants in three French catchments. Environmental Science and Pollution Research (8):5267-81. doi:10.1007/s11356-013-2396-0

Government of Canada, 2015. Intensity-Duration-Frequency (IDF) Files [WWW Document]. URL ftp://ftp.tor.ec.gc.ca/Pub/Engineering_Climate_Dataset/IDF/ (accessed 11.15.16).

Hatt, B.E., Fletcher, T.D., Deletic, A., 2009. Hydrologic and pollutant removal performance of stormwater biofiltration systems at the field scale. Journal of Hydrology 365, 310-321. doi:10.1016/j.jhydrol.2008.12.001

Heasom, W., Traver, R.G., Welker, A., 2006. Hydrologic Modeling of a Bioinfiltration Best Management Practice. Journal of the American Water Resources Association 42, 1326-1347.

Kazemi, F., Beecham, S., Gibbs, J., 2011. Streetscape biodiversity and the role of bioretention swales in an Australian urban environment. Landscape and Urban Planning 101, 139-148. doi:10.1016/j.landurbplan.2011.02.006

Kottek, M., Grieser, J., Beck, C., Rudolf, B., Rubel, F., 2006. World Map of the Köppen-Geiger climate classification updated. Meteorologische Zeitschrift 15, 259263. doi:10.1127/0941-2948/2006/0130

Le Coustumer, S., Fletcher, T.D., Deletic, A., Barraud, S., Poelsma, P., 2012. The influence of design parameters on clogging of stormwater biofilters: A large-scale column study. Water Research 46, 6743-6752. doi:10.1016/j.watres.2012.01.026

LeFevre, G., Paus, K.H., Natarajan, P., Gulliver, J.S., Novak, P.J., Hozalski, R.M., 2014. Review of Dissolved Pollutants in Urban Storm Water and Their Removal and Fate in Bioretention Cells. Journal of Environmental Engineering 141.

Le Nouveau, N., 2010. Les multiples vertus des jardins de pluie.

Leroy, M.-C., 2015. Etude intégrée de l'abattement en contaminants dans un système Eau/Sol/Plante. Problématique des aménagements de voiries urbaines. Université de Rouen.

Leroy, M.-C., Marcotte, S., Moncond'huy, V., Legras, M., Portet-Koltalo, F., Le Derf, F., 2016. Epuration des eaux de ruissellement dans les noues de voirie: le rôle du sol et des plantes, in: NOVATECH 2016.

Liu, J., Sample, D., Bell, C., Guan, Y., 2014. Review and Research Needs of Bioretention Used for the Treatment of Urban Stormwater. Water 6, 1069-1099. doi:10.3390/w6041069

MGD Infiltration, 2006. Guide Technique: Recommandations pour la faisabilité, la conception et la gestion des ouvrages d'infiltration des eaux pluviales en milieu urbain.

NCDWQ (North Carolina Division of Water Quality), 2017. Stormwater Design Manual.

NIWA, 2016. High Intensity Rainfall System V3 [WWW Document]. URL https://hirds.niwa.co.nz/ (accessed 12.5.16).

NSC (North Shore City), Malcolm, M., Lewis, M., 2008. North Shore City Bioretention Guidelines.

Peel, M.C., Finlayson, B.L., McMahon, T.A., 2007. Updated world map of the Köppen-Geiger climate classification. Hydrology and Earth System Sciences 11, 1633-1644. doi:10.5194/hess-11-1633-2007

PGCDER (The Prince George's County Department of Environmental Resources), 2007. Bioretention Manual.

Préfet des Bouches-du-Rhône, 2015. Principes de gestion des eaux pluviales dans les projets d'aménagement dans le Bouches du Rhône [WWW Document]. URL http://www.bouches-du-rhone.pref.gouv.fr/content/download/17292/107837/file/doctrine2150.pdf (accessed 12.5.16).

The Prince George's County, Maryland, 1999. Low-Impact Development Design Strategies.

PUB (Public Utility Board), 2011. Engineering Procedures for ABC Waters Design Features.

Qingyu, Z., Jiangcheng, H., Mui, H.A., 2012. A simple rainfall intensity formula for Singapore. The IES Journal Part A: Civil \& Structural Engineering 5, 62-64. doi:10.1080/19373260.2012.634252

Ramier, D., Caupos, E., Branchu, P., Dubois, P., Georgel, P., Neveu, P., Paupardin, J., Ratovelomanana, T., Saad, M., Seidl, M., Thomas, E., Viau, J.-Y., Gromaire, M.-C., 2016. Mesurer l'efficacité des techniques alternatives pour la maîtrise des flux polluants: un challenge métrologique. Le cas de quatre dispositifs innovants suivis dans le cadre du projet ROULEPUR, in: NOVATECH 2016. Presented at the Novatech.

Roy-Poirier, A., Champagne, P., Filion, Y., 2010. Review of bioretention system research and design: past, present, and future. Journal of Environmental Engineering 136, 878-889.

Schlüter, W., Jefferies, C., 2005. The real issues with in-ground SUDS in Scotland, in: 10th International Conference on Urban Drainage.

Servier, A., 2016. Les Noues: Conception et réalisation.

Thomas, A.O., Bos, D., Morison, P., Fletcher, T.D., 2016. Operation and Maintenance of Stormwater Control Measures, in: NOVATECH 2016.

UDFCD (Urban Drainage and Flood Control District), 2015a. Bioretention, in: Urban Storm Drainage Criteria Manual: Stormwater Quality. Denver, Colorado.

UDFCD (Urban Drainage and Flood Control District), 2015b. Calculating the WQCV and Volume Reduction, in: Urban Storm Drainage Criteria Manual: Stormwater Quality. Denver, Colorado.

US Department of Commerce, N., 2016. Precipitation Frequency Data Server: Contiguous US [WWW Document]. URL http://hdsc.nws.noaa.gov/hdsc/pfds/pfds_map_cont.html?bkmrk=il (accessed 10.18.16).

UWWRI (University of Wisconsin Water Resources Institute), Atchison, D., Potter, K., Severson, L., 2006. Design Guidelines for Stormwater Bioretention Facilities.

Zgheib, S., Moilleron, R., Chebbo, G., 2012. Priority pollutants in urban stormwater: Part 1 - Case of separate storm sewers. Water Research 46, 6683-6692. doi:10.1016/j.watres.2011.12.012 